Nikotin als Ursache der Sucht ist zweifelsfrei belegt. Die krankmachende Wirkung des Zigarettenrauchens betrifft nebst Krebs weitere tödliche und invalidisierende Erkrankungen wie KHK und COPD. Rauchen verursacht in jedem Lebensalter Krankheiten, von der Mangelgeburt bei Rauchen während der Schwangerschaft bis zur invalidisierenden Atherosklerose mit Beinamputation des vorzeitig alternden Rauchers. Letztlich stirbt jeder zweite Raucher wegen dieser Sucht frühzeitig. Bei uns sind es zunehmend Kinder, Jugendliche und vor allem Frauen, die nun im Visier der Tabakgiganten sind.

Sollten die jetzigen Trends der Zigarettensucht anhalten, wird in diesem Jahrhundert die durch Zigarettenrauchen umgebrachte Anzahl Menschen eine Milliarde betragen. Ab dem Jahr 2050 würden pro Jahrzehnt 100 Mio. Menschen sterben.
In der westlichen Welt haben vor allem die US-Bundesstaaten Kalifornien und Massachusetts gezeigt, dass konsequente und anhaltende Bemühungen in der Bekämpfung des Rauchens im öffentlichen Bereich zur deutlichen Reduktion des Zigarettenkonsums führen können und solche Resultate nachhaltig sind. Dies ist auch bei uns möglich, wenn die Mittel und flankierenden politischen Massnahmen dafür beschlossen werden.

Wir als Spezialistinnen und Spezialisten haben hier eine Verantwortung von grosser Tragweite, da es sich um die grösste vermeidbare Krankheitsursache mit Todesfolge in unserem Land handelt.

Prof. Dr. Thomas Cerny, Präsident der Krebsliga Schweiz

\title{
Resolution für eine rauchfreie Zukunft
}

\author{
Nationale Tabakpräventionskonferenz vom 1. und 2. Dezember 2004 in Freiburg
}

\section{Schritte auf dem Weg in eine rauchfreie Zukunft}

Jeden Tag sterben in der Schweiz mehr als 20 Menschen vorzeitig an den Auswirkungen des Rauchens. Dies sind jährlich über 8000 Personen. Zusätzlich verursacht das Passivrauchen pro Jahr Hunderte von weiteren Todesopfern. 45 Prozent der tabakbedingten Todesfälle werden ausgelöst durch Herz-Kreislauf-Krankheiten, 25 Prozent durch Lungenkrebs, 18 Prozent durch Atemwegserkrankungen und 12 Prozent durch andere Krebsarten.

Die Teilnehmerinnen und Teilnehmer der Nationalen Tabakpräventionskonferenz vom 1. und 2. Dezember 2004 in Freiburg setzen sich deshalb für folgende Massnahmen ein:

\section{Schutz vor Passivrauchen}

Menschen, die rauchfrei leben wollen, werden nur dann tatsächlich vor Passivrauchen geschützt, wenn die Luft in Innenräumen keinen Tabakrauch enthält. Die Zeit der freiwilligen Einschränkungen oder der kleinen Nichtraucherecken ist vorbei.

Wir begrüssen folglich die politische Initiative des Tessiner Staatsrats, öffentliche Räume grund- sätzlich rauchfrei zu gestalten. Die übrigen Kantone werden aufgefordert, den Schutz vor Passivrauchen ebenfalls gesetzlich zu gewährleisten.

Das Recht auf einen rauchfreien Arbeitsplatz soll ohne Vorbehalt gelten. Die Verordnung 3 zum Arbeitsgesetz ist entsprechend anzupassen. Die kantonalen Arbeitsinspektorate haben ihre Kontrollfunktion wahrzunehmen.

\section{Unterstützung beim Rauchstopp}

Die Mehrheit der Raucherinnen und Raucher will von der Nikotinabhängigkeit loskommen. Die Massnahmen der nationalen Rauchstoppkampagne «Rauchen schadet - Let it be» müssen fortgesetzt werden. Ärztinnen und Ärzte, die Teams der zahnmedizinischen Praxen, die Apotheken und die kantonalen Gesundheitsfachstellen sind $\mathrm{zu}$ motivieren und $\mathrm{zu}$ befähigen, Raucher und Raucherinnen beim Rauchstopp zu beraten und zu unterstützen.

Diese Arbeit der Fachleute muss entsprechend honoriert werden. Die obligatorische Krankenpflegeversicherung hat für eine beschränkte Dauer die Kosten zu übernehmen für Medikamente, die erwiesenermassen die Erfolgsquoten des Rauchstopps verbessern. 


\section{Jugendliche vom Tabakkonsum abhalten}

Die Zigarette ist das einzig legal erhältliche Produkt, bei dessen Verwendung gemäss Anweisungen der Hersteller die Hälfte der Konsumentinnen und Konsumenten vorzeitig stirbt. Aus diesem Grund fordern wir eine tabakfreie Werbung. Heute können die Zigarettenhersteller in der Schweiz ohne grosse Einschränkungen ihre Produkte durch Werbung und Promotion anpreisen.

Teure Zigaretten führen zu einem Konsumrückgang, besonders bei Jugendlichen. Doch verglichen mit den umliegenden Ländern sind Zigaretten in der Schweiz immer noch zu billig.

Zudem ist der Zugang zu Tabakwaren, gerade für Jugendliche, zu erschweren. Zigaretten werden weiterhin an mehr Verkaufsstellen angeboten als Brot oder Milch.

Bildungsanstalten haben einen wichtigen Auftrag in der Gesundheitsförderung zu erfüllen. Rauchfreie Schulen können diese Bemühungen verstärken.

\section{Der Tabakindustrie entschlossen entgegentreten}

Die Rahmenkonvention der Weltgesundheitsorganisation zur Tabakkontrolle (FCTC), die im Juni 2004 von der Schweiz unterzeichnet wurde, schafft die Grundlage zur landesweiten Umsetzung der vorgeschlagenen Massnahmen:

- rauchfreie öffentliche Räume;

- Schutz vor Passivrauchen auch am Arbeitsplatz;

- Unterstützung beim Rauchstopp;

- hohe Zigarettenpreise;

- Werbeverbot für Tabakprodukte;

- Beschränkung der Erhältlichkeit von Tabakwaren, besonders für Jugendliche;

- rauchfreie Schulen.

Wir fordern Bundesrat und Parlament auf, die Rahmenkonvention zur Tabakkontrolle noch in dieser Legislatur zu ratifizieren. Zur Stärkung der Förderung des Nichtrauchens ist eine strenge Regulierung der Tabakprodukte notwendig. 